\title{
Role of Physical Activity in the Management of Cancer
}

\author{
BEN OHURUOGU (PH.D) \\ Department Of Human Kinetics And Health Education, Ebonyi State University, Abakaliki. \\ ALOH CHINYERE ADA \\ Faculty of Educaton, Alex Ekweme Federal University, Ikwo \\ ALOR ROSELINE CHINYERE \\ Physical And Health Education Department Ebonyi State College Of Education, Ikwo
}

\begin{abstract}
This paper focuses on the role of physical activity in the management of cancer. It looked at the potential role of physical activity during and after cancer treatment. The paper X-rayed patients receiving cancer treatment, patients who have finished treatment and recovering from physical function. And also looked at patients after diagnosis reducing the risk of cancer recurrence and mortality and the risk of developing other conditions. And finally looked at patients with advanced cancer cases and how they can be helped to maintain independence and well-being towards the end of their life. It however, recommended among others that stakeholders in cancer management should form mechanisms within the cancer care period to support people in maintaining or initiating physical activity during and after treatment.
\end{abstract}

Keywords: Cancer, Mortality, Patients, Physical Activity.

DOI: $10.7176 / \mathrm{JEP} / 10-29-14$

Publication date:October $31^{\text {st }} 2019$

\section{INTRODUCTION}

Physical activity has been defined as any body movement produced by skeletal muscles that results in energy expenditure. However, the complexities of the numerous forms and etiologies of cancers provide researchers with difficult problems in identifying links with exercise. Nevertheless, there is growing evidence that has revealed or supported the role of physical activity during and after cancer treatment. Physical activity is important for patients at all stages of cancer problem.

According to Speck, Courueya, Masses, Dural and Schoritz (2010), physical activity has proved to improve or prevents the decline of physical function without increasing fatigue, it has also helped to recover physical function, helped to reduce the risk of cancer recurrence and mortality, for some cancer and long term conditions and also help maintain independence and well-being,

There are over two million people living with cancer or beyond cancer in the world and if the current trends continue, there will be more than four million people living with or beyond cancer by the year 2030 (Mcneely, Campbell, Ospina, 2010). Many cancer survivors experience long lasting adverse effects of their diseases and treatments. These problems include fatigue weight changes, urinary and bowel problems, peripheral neuropathy, osteoporosis, cardio-toxicity, lymphaedema, heart flushes, might sweats, anxiety, depression and difficulty with memory concentration (camp and Daniel, 2008). A survey conducted by Macmillian cancer support indicated 78\% of people living after cancer experienced at least one of those problem while $71 \%$ experienced at least one of these more than ten years after treatment (Speck, Courneya, Masse, Duval and Schmitz, 2010).

\section{Potential role of Physical Activity in the Management of Cancer}

There is strong evidence to support the role of physical activity during and after cancer treatment. Keeping active throughout the cancer period can preserve or improve physical function and psychological well-being. It can also help reduce the negative impact of some cancer related side effects. Regular physical activity according to Mess, Wall, Oakes, Robison, and Gurney (2006) also has potential role in reducing risk of cancer recurrence and increasing survival. Promoting physical activity as part of cancer care is a cost-effective intervention for care givers. This is true because routine provision of physical activity and exercise rehabilitation services within the cancer care pathway will enable the stakeholders in the crusade to stop the menace of cancer, maximize the improvements to quality of care and patients outcomes.

\section{Patients Receiving Cancer Treatment-Impacts on Physical Function}

Most cancer treatments are associated with a range of side effects. Patients typically lose cardiovascular and muscular fitness, experience fatigue and report diminished psychological well-being over the course of treatment. Additionally, many are advised to rest through treatment. An over emphasis on energy conservation have been known to exacerbate the treatment related losses of physical conditioning and muscle strength. This has proved to 
add to the development of a perpetual cycle of deteriorating function and accumulating fatigue that is very hard to overcome (Ibrahim, and Al-Homaidh, 2010). Current evidence supports the recommendation of exercise during treatment to prevent decline in functional outcomes without increasing fatigue. Exercise has also been proved to have positive effects on aspects of psychological well-being, such as anxiety and self-esteem.

\section{Patients who have Finished Treatment Recovering from Physical Function}

Following the completion of cancer treatment, many patients continue to experience adverse effects of treatments. There is sufficient evidence to recommend exercise as an effective way to help recover physical function; including building muscle strength and improving cardiovascular fitness, manage fatigue, improve quality of life and mental health, and control body weight (Holick, Newcomb and Trentham-Dietz, 2008).

\section{Risk of Cancer Recurrence and Mortality}

In addition to the physical and mental health benefits achieveable through exercise cancer diagnosis can influence disease recurrence and mortality for some cancers (Patterson, Cadmus, Emond and Pierce, 2010). Although the body of evidence on the survival benefits associated with physical activity is still developing and research to data in this area is highly encouraging.

\section{Patients following diagnosis-reducing the risk of Developing other Conditions}

Lack of physical activity is a risk factor for several major chronic diseases including coronary heart disease, stroke, diabetes mellitius, esteoporosis, obesity, some cancers and dementia. Risk reductions of 20-50\% are possible for people who are active at the recommended levels relative to those who are inactive (Chan, Lui, and So, 2010), The benefits of exercise for people who are living with and beyond are deor. Yet regular and sustained physical activity is also important for reducing the risk of developing other long-term conditions or new cancers. Equally, important is the role of physical activity in helping to manage existing chronic conditions in cancer survivors. A study carried out by Macmillam (2009) showed that just under half (49\%) of cancer survivors have at least one other chronic conditions. Prevalence of co-morbidities increase with age, and a study conducted by Mutrie and Campbell (2007) on co-morbidities in cancer survivors age 70yrs and above reported an average of three co-morbidities in cancer survivors considerable evidence supports a beneficial role of physical activity in helping the long-term management of cardiovascular diseases, musculoskeletal health conditions, and mental illness (Stone, Richardson, Ream, Smith, Kerr and Kearnery, 2000)

\section{Patients with advanced cancer-helping maintain independence and well-being toward end of life.}

There is accumulating evidence of the benefits of exercise for patients with advanced cancer. Systematic reviews of six studies in palliative care setting and eight studies of patients with metastatic cancer concluded exercise was feasible for these patients and offered important benefits in physical functioning symptoms, and quality of life (Holmes, 2005). Patients preference is important to consider, but exercise can be recommended to help maintain functional independence and quality of life towards the end of life. The write up in this paper demonstrate that good evidence exists to support the promotion of physical activity throughout the cancer care period. In conclusion, activities should be designed for patients at all stages of cancer from initial diagnosis through to the later stages where activity can continue to benefit physical function and quality of life. The evidence shows that if an activity recommendation is carefully tailored to the individual and takes account of potential side effects, it is likely to have a positive impact.

\section{Strategies for Preventing Cancer}

Strategies for preventing cancer includes avoiding tobacco, eating a varied, moderate diet and controlling weight; protecting the skin from the sun, avoiding exposure to environmental and occupational carcinogens, staying alert for cancer warning signs and exercising regularly.

\section{Recommendations}

The following recommendations are made

1. Cancer survivors should be advised to gradually build up to health- related physical activity guidelines for the general population. The point is that if any activity recommendation is carefully tailored to the individual, it is likely to have a positive impact on the patients.

2. Stakeholders should form mechanisms within the cancer care period to support people in maintaining or initiating physical activity during and after treatment.

\section{References}

Chan, D.N.S., Lui, L.Y.Y. and So W.K.W. (2010). Effectiveness of exercise programmes on shoulder mobility and lymphoedema after axuillary lymph node dissection for breast cancer: systematic review. Journal of 
Advanced Nursing 66(9): 1902-1914.

Gramp and Daniel (2008). Physical activity for the management of cancer-related fatigue in adults. Cochrane. Database system Rev.

Holick, C.N., Newcomb, P.A. Trentham-dietz A. (2008). Physical activity and survival after diagnosis of invasive breast cancer, cancer Epidemiology Glomakers preview: 17:379-386.

Holmes M.D. (2005). Physical activity and survival after breast cancer diagnosis. Jama 293 (20). 2479-86.

Ibrahim, E.M. and Al-Homaidh A. (2010). Physical activity and survival after breast-cancer diagnosis: Metaanalysis of published studies. Medicine oncology (Epublished ahead print)

Lee, I. M. (1994) Physical activity, fitness, and cancer in C, Bonchard, R, J. Shephard and T. Stephens (eds) Physical activity, fitness and health $(814-831)$ Champaign, 111, Human Kinetics

Mcneedy, M.L., Campbell, K., Ospina, M., Rowe, B.H., Dabbs, K., Klassen, T.P., Mackey, J. and Courneya, K. (2010). Exercise interventions for upper limb dysfunction due to breast cancer treatment. Cochrance database of systematic reviews. 6:2.

Mess, K.K., all, M.M., Oakes, M. Robinson, L.L., Gurney, J.G. (2006). Physical performance limitation and participation restrictions among cancer survivors: A population based study. Annals of epidermiology 16:197-205.

Mutrie, Campbell (2007). Benefits of supervised group exercise programmes for women being treated for early stage breasts cancer: Pragmatic randomized controlled trial. British Medical Journal 334:517

Patterson, R.E., Cadmua, L.A., Edmond, J.A and Pieve J.P. (2010). Female breast cancer prognosis: A review of the epidemiologic literature. $66: 5-15$

Speck, R.M., Courneya, K.S., Masse, L.C. Duval, S. and Schmitz, K.H. (2010). :An update of controlled physical activity trials in cancer survivors: A systematic review and meta-analysis. Journal cancer survival 4:87-100.

Stone, P. Richardson, A. Ream, E, Smith, A.G., Jerrm DJ and Kearney N. (2000). Cancer related fatigue: Inevitable, unimportant and untreatable. Results of a multi-centre patient survey. Annual oncology 11: 971-975. 